\title{
Drug development: Lessons from nature (Review)
}

\author{
SUNIL MATHUR $^{1}$ and CLARE HOSKINS ${ }^{2}$ \\ ${ }^{1}$ Molecular Drug Research Laboratory, Edinburgh Napier University, Edinburgh EH11 4BN; \\ ${ }^{2}$ Institute of Science and Technology in Medicine, Keele University, Hartshill, Stoke-on-Trent ST4 7QB, UK
}

Received August 25, 2016; Accepted December 14, 2016

DOI: $10.3892 /$ br.2017.909

\begin{abstract}
Natural products have been acknowledged for numerous years as a vital source of active ingredients in therapeutic agents. In particular, the use of active ingredients derived from plants for use in microbial natural products have long been used before the dawn of modern medicine. From ancient times, the efficacy of natural products has been associated with the chemistry, biochemistry and synthetic activities of natural products. Thus, with scientific advancement in modern molecular and cellular biology, analytical chemistry and pharmacology, the unique properties of these natural products are being harnessed in order to exploit the chemical and structural diversity and biodiversity of these types of products in relation to their therapeutic effect. Often, new molecules of interest in drug design units focus on the rearrangement of chemical entities or structural isomers of naturally occurring products in order to generate new molecules; these may be formulated into clinically useful therapies.
\end{abstract}

\section{Contents}

1. Introduction

2. Sources of drugs

3. Current uses of natural product drugs

4. Natural product drug development

5. Future directions for natural product drug discovery

6. Conclusions

\section{Introduction}

A natural product is a chemical entity, formed by a naturally occurring living organism with pharmacological properties, which may contribute to vital drug discovery and design. The

Correspondence to: Dr Sunil Mathur, Molecular Drug Research Laboratory, Edinburgh Napier University, Sighthill Campus, 9 Sighthill Court, Edinburgh EH11 4BN, UK

E-mail: s.mathur@napier.ac.uk

Key words: natural products, drug discovery, combinatorial chemistry, high throughput screening crude substance extracted from the body of medicinal plants, animals, microbes or microorganism fermentation broths contains unique and structurally diverse chemical components. Natural products have been vital in pharmaceutical and biotechnology industries, as a vast range of modern medicines are based upon either naturally occurring molecules, or derivatives of these. Generally, the therapeutic agents that are inhaled, ingested and injected are a mixture of complex therapeutic compounds.

\section{Sources of drugs}

The complexity of the therapeutic agents depends on the mixture of chemicals prepared synthetically. Therapeutic agents are considered as natural, synthetic, or semi-synthetic dependent on the source from which they were generated (1). The natural environment remains a significant origin of novel therapeutic agent compounds. Natural therapeutic agents are prepared from compounds found occurring in nature, which contain active components in extract form created from sources, including plants, microbes, minerals and animals. The most dominant natural medicine source is plants, due to their chemical and structural diversity and the biodiversity of their components. Examples of medicines that are derived from plants are aspirin (from willow tree bark) (2), digoxin (from the flower, Digitalis lanata) (3) and morphine (from opium) (4). Indeed, it is projected that in the industrialized nation, $>60 \%$ of all medicines are either natural products or secondary metabolites thereof (5). Although there are challenges from different, novel drug discovery methods, natural products continue to produce additional clinical candidates and medicinal compounds. These compounds remain an important foundation of novel medicines, particularly in anti-infective [azitromycin (6) and amphotericin B (7), acyclovir (8) and romidepsin] (9) therapies. Many have subsequently advanced into clinical trials or onto the market (4). The expansion of novel technologies (high throughput screening, automated separation methods and combinatorial chemistry) in drug discovery has transformed the screening of natural products due to their chemical and structural diversity and biodiversity (10).

The distinctive structural diversity of natural compounds is advantageous during the production of combinatorial libraries using semi-synthetic methods. Semi-synthetic therapeutic agents are a hybrid of natural and synthetic sources. Semi-synthetic therapeutic agents are generally produced 
by transforming starting materials from natural sources into final products via chemical reactions (11). Examples of semi-synthetic medicines are antibiotic penicillin (12) and paclitaxel (an anticancer drug derived from the Pacific yew tree, Taxus brevifolia) (13).

\section{Current uses of natural product drugs}

The importance of natural products used in the treatment and prevention of human diseases may be evaluated by the following: The introduction of novel, chemical, diverse entities with their application as patterns for semi-synthetic and total synthetic modification; the quantity of diseases treated by these diverse compounds; and their rate of use in the treatment of diseases (14). Natural products continue to be the focus of pharmaceutical research, due to the failure of substitute drug discovery methods to provide many lead compounds in vital therapeutic areas, such as anti-infective and metabolic diseases. Research into natural products continues to provide a novel template for drug discovery of lead compounds for the pharmaceutical industry. Currently, it is undisputed that natural products have been, and will be, key sources of innovative pharmaceutical therapeutic agents. The drug metabolites produced from synthetic drug sources exert fewer therapeutic effects with adverse side effects. Synthetic therapeutic agents are comprised of small chemical building blocks, which are not found in nature, but are produced with the help of computer-based research, such as computational chemistry and using various chemical sources, such as arterolane (anti-malaria drug) (15). However, therapeutic agents formed from natural sources may avoid the side effects, as they produce physiological and pharmacological effects within living cells. In addition, natural products have a wider distribution of molecular properties, such as lower molecular mass, partition coefficient and structural diversity (16). Moreover, natural products have more interaction with proteins, enzymes and other biological molecules. Furthermore, natural products contain fewer heavy metals and have greater molecular rigidity when compared with synthetic compounds and combinatorial libraries (13).

\section{Natural product drug development}

Although natural products have been vital in drug discovery, recently pharmaceutical companies have either terminated or substantially reduced their natural product research (13). The reason for this is the development of advanced techniques, such as metagenomics, combinatorial chemistry and high throughput screening. Furthermore, not all natural products can be completely synthesized, due to their structural complexity and as the process would be time consuming and expensive. However, with the help of advanced drug discovery and increased medicinal chemistry knowledge, the pharmaceutical and biotech industries are once again turning to natural products in order to harness new useful entities or derivatives of these in therapeutics. Often, this is driven by therapeutic need in difficult to treat diseased states or clinical conditions such as cancer, obesity and infection activated by multi-resistant pathogens. Plants, microorganisms, fungi and bacteria have been invaluable in drug design and continue to be a rich source of natural products in the drug discovery process. The war against various multi-resistant pathogens requires advanced and more effective therapeutic agents. Only $5-15 \%$ of terrestrial plants out of the existing 250,000 species have been chemically and pharmacologically scrutinized in resourceful mode for use as therapeutic agents. Furthermore, the microbial domain signifies $90 \%$ of all natural diversity, however $<1 \%$ has been discovered (13). Currently, the threat of extinction of natural diversity due to environmental reasons, such as global warming and toxic waste (including heavy metals and cyanides) from synthetic compounds and multidrug resistance to traditional medicines is high. Therefore, it is considered to be particularly important to establish the importance of natural diversity and bioresources to educate current research strategies in biotechnological and large pharmaceutical companies for use in the drug discovery process.

The objective of all drug discovery processes is to establish the most promising lead compounds, which may be used as a therapeutic agent and facilitated with treating medical conditions, including infections, cancer, nervous system diseases, high blood pressure and metabolic diseases (13). During the early stages of the drug design process, scientists must isolate and purify the lead compounds from their natural source by utilizing various methods depending on the structural diversity, stability and quantity of the compound required. High throughput screening has been used to screen the lead compounds against specific targets. Subsequent to vital biochemical and pharmacological tests, the promising compounds for the specific targets are selected. At this phase in the drug design process, many lead compounds are not selective enough for their target molecule. In a bid to improve their selectivity, scientists modify the lead compound structures as per the expected structure-activity relationships. If the modifications increase the selectivity, the promising compounds move to in vitro and in vivo testing in designated disease facsimiles (15). Upon a positive result, scientists are required to conduct safety tests on the selected compounds to establish the mechanism by which the drug is absorbed, distributed, metabolized and excreted. This process (termed pharmacokinetics) indicates the mechanisms that occur when the therapeutic agent enters the body. If all of the results and optimizations are positive, then lead compounds may become potential candidate drugs (13).

\section{Future directions for natural product drug discovery}

Biological diversity and modern genomics are crucial topics leading bioscience in the 21 st century, and biodiversity is considered to be increasingly important in this field (17). Indeed, natural products are full of biodiversity, the research of which requires extensive and in-depth experience, particularly in taxonomy, which takes time to accrue. Pharmaceutical and biotechnology companies must adapt to the immediate need for new medicines and adopt new techniques in order to overcome clinical conditions that cannot be treated using conventional therapies. Hence, looking into the structure-activity relationships of naturally occurring active ingredients may offer a strategy to overcome such medicinal challenges. By contrast, if industries want to contribute to, and take advantage of, the drug discovery process, then they must establish techniques, such as 
computational chemistry, to achieve more sustainable use of their natural resources. Industries must realize that although natural products perform important biological functions with a valuable role in the ecosystem, their economic value may be less until their primary utility is exposed and established. To understand the utility and important benefits, pharmaceutical industries must be prepared to contribute actively and financially to the drug discovery process (13). Natural products are considered to be particularly valuable for the production of various precious lead components ranging from simple chemical structures to highly complex structures. Natural metabolites are always superior in terms of biochemical and pharmacological activities when compared with secondary metabolites (18). The current drug discovery processes arising from natural resources is primarily focused on the isolation, purification, screening and discovery of novel drug candidates. In order to move forward with any potential lead compounds, large scale extraction or biotechnology production is required in order to move the lead compounds forward and make them clinically feasible.

In the revolutionized and highly competitive field of modern pharmaceutical research, natural products can mimic distinctive metabolites of biological functionality such as hormones and naturally occurring ligands (19) as well as possessing great structural diversity, which is necessary for the drug discovery process. To regenerate the interest of the pharmaceutical industry and, in order to be competitive with other advanced drug discovery techniques, natural product research must constantly increase the efficiency of screening (computational or otherwise), isolation, robotic separation and purification. Additionally, drug stability and formulation studies as well as efficacy, pharmacokinetics and metabolic engineering investigations are required in order to identify compounds of interest and move them forward into clinically applicable therapies. The growing demand for medicinal plants in traditional and herbal medicine is also threatening their existence. To improve biodiversity across the globe, it is critical to cultivate medicinal plants using controlled and scientific methods, to provide the desired medicinal plants at high quality. In addition, toxic waste from the production process greatly impacts the biological ecosystem; it has particularly harmful effects on the plants and aquatic life in streams (20). To protect the natural resources, toxic waste containing toxic elements and unpleasant, odor-producing chemicals must be separated and treated independently. This protection of the natural flora will aid in the sustainability of ecosystems and hence protect any natural resource that may be used for the discovery of unique medicinal and biological metabolites.

\section{Conclusions}

An integrative approach of coupling advanced genetic sequencing with the management of biosynthetic pathways, may deliver a sustainable and realistic route for the future discovery of pharmaceutical drug candidates. The success of natural products in past and present drug discovery is fundamentally associated with their complex structural diversity, as well as advancement in understanding of how structural confirmations or functionality contribute to molecular activity in biomedicine. Nature continues to provide biological diversity, which is important to target, particularly considering the insufficient results obtained from combinatorial techniques. It is hoped that the environment will continue to provide undiscovered resources, permitting the unique discovery of novel weapons against developing infections.

\section{References}

1. Feher M and Schmidt JM: Property distributions: Differences between drugs, natural products, and molecules from combinatorial chemistry. J Chem Inf Comput Sci 43: 218-227, 2003.

2. Li J, Larregieu CA and Benet LZ: Classification of natural products as sources of drugs according to the biopharmaceutics drug disposition classification system (BDDCS). Chin J Nat Med 14: 888-897, 2016.

3. Gerwick WH: Plant sources of drugs and chemicals. In: Encyclopedia of Biodiversity. 2nd edition. Elsevier Inc. pp129-139, 2013.

4. Butler MS: The role of natural product chemistry in drug discovery. J Nat Prod 67: 2141-2153, 2004.

5. Eddershaw PJ, Beresford AP and Bayliss MK: ADME/PK as part of a rational approach to drug discovery. Drug Discov Today 5: 409-414, 2000.

6. Bérdy J: Thoughts and facts about antibiotics: Where we are now and where we are heading. J. Antibiotics (Basel) 65: 385-395, 2012.

7. Gao Y, Deng K, Cao Z, Graziani EI, Gilbert AM, Koehn FE, Wood A, Doherty P and Walsh FS: Amphotericin B, identified from a natural product screen, antagonizes CNS inhibitors to promote axon growth via activation of an Akt pathway in neurons. J Neurochem 113: 1331-1342, 2010.

8. Zhang L and Demain AL (eds): Nautral Products: Drug Discovery and Therapeutic Medicine. Humana Press Inc., Totowa, NJ, pp3-29, 2005.

9. Mottamal M, Zheng S, Huang TL and Wang G: Histone deacetylase inhibitors in clinical studies as templates for new anticancer agents. Molecules 20: 3898-3941, 2015.

10. Patwardhan B, Vaidya ADB and Chorghade M: Ayurveda and Traditional Chinese Medicine: A Comparative Overview. Curr Sci Assoc/Indian Academy of Sciences 86: 789-799, 2004.

11. Cragg GM and Newman DJ: Natural products: A continuing source of novel drug leads. Biochim Biophys Acta 1830: 3670-3695, 2013

12. Oshiro BT: The semisynthetic penicillans. Prim Care Update Ob Gyns 6: 56-60, 1999.

13. Lahlou M: The Success of Natural Products in Drug Discovery. Pharmacol Pharm 4: 17-31, 2013.

14. Chin YW, Balunas MJ, Chai HB and Kinghom AD: Drug Discovery from Natural Sources. AAPS J 8: 239-242, 2006.

15. Valecha N, Looareesuwan S, Martensson A, Abdulla SM, Krudsood S, Tangpukdee N, Mohanty S, Mishra SK, Tyagi PK, Sharma SK, et al: Arterolane, a new synthetic trioxolane for treatment of uncomplicated Plasmodium falciparum malaria: A phase II, multicenter, randomized, dose-finding clinical trial. Clin Infect Dis 51: 684-691, 2010.

16. Pascolutti M and Quinn RJ: Nautral producsts as lead structures: chemical transformations to create lead-like libraries. Drug Discov Today 19: 215-221, 2014.

17. Cordell GA: Biodiversity and drug discovery - a symbiotic relationship. Phytochemistry 55: 463-480, 2000.

18. Atanasov AG, Waltenberger B, Pferschy-Wenzig EM, Linder T, Wawrosch C, Uhrin P, Temml V, Wang L, Schwaiger S, Heiss EH, et al: Discovery and resupply of pharmacologically active plant-derrived natural products: A review. Biotechnol Adv 33: 1582-1614, 2015.

19. Ji HF, Li XJ and Zhang HY: Natural products and drug discovery. Can thousands of years of ancient medical knowledge lead us to new and powerful drug combinations in the fight against cancer and dementia? EMBO Rep 10: 194-200, 2009.

20. Shaban NS, Abdou KA and Hassan N E-HY: Impact of toxic heavy metals and pesticide residues in herbal products. Beni-Suef University Journal of Basic and Applied Sciences 5: 102-106, 2016. 